\title{
HS-SPME Combined with GC-MS/O to Analyze the Flavor of Strong Aroma Baijiu Daqu
}

\author{
Zhe Wang ${ }^{1,2,+}$, Song Wang ${ }^{1,2,+}$, Pengfei Liao ${ }^{1,2}$, Lu Chen ${ }^{1,2}$, Jinyuan Sun ${ }^{1,2, * \mathbb{D}}$, Baoguo Sun ${ }^{1,2}$, Dongrui Zhao ${ }^{1,2}$, \\ Bowen Wang ${ }^{1,2}$ and Hehe $\mathrm{Li}^{1,2}$
}

1 Key Laboratory of Brewing Molecular Engineering of China Light Industry, Beijing Technology and Business University, Beijing 100048,China; 1930201035@st.btbu.edu.cn (Z.W.); wangs_2278@126.com (S.W.); liaopfxs@163.com (P.L.); chenlu_btbu@163.com (L.C.); sunbg@btbu.edu.cn (B.S.); zdr@btbu.edu.cn (D.Z.); wangbw@btbu.edu.cn (B.W.); lihehe@btbu.edu.cn (H.L.)

2 Beijing Laboratory for Food Quality and Safety, Beijing Technology and Business University, Beijing 100048, China

* Correspondence: sunjinyuan@btbu.edu.cn; Tel./Fax: +86-010-6898-4890

+ These authors contributed equally to this work.

check for updates

Citation: Wang, Z.; Wang, S.; Liao, P.; Chen, L.; Sun, J.; Sun, B.; Zhao, D.; Wang, B.; Li, H. HS-SPME Combined with GC-MS/O to Analyze the Flavor of Strong Aroma Baijiu Daqu. Foods 2022, 11, 116. https://doi.org/ 10.3390 /foods 11010116

Academic Editor: Onofrio Corona

Received: 18 November 2021

Accepted: 30 December 2021

Published: 3 January 2022

Publisher's Note: MDPI stays neutral with regard to jurisdictional claims in published maps and institutional affiliations.

Copyright: (C) 2022 by the authors. Licensee MDPI, Basel, Switzerland. This article is an open access article distributed under the terms and conditions of the Creative Commons Attribution (CC BY) license (https:// creativecommons.org/licenses/by/ $4.0 /)$.

\begin{abstract}
Daqu has gained wide attention because it is an essential source of microorganisms and flavor in baijiu production. In this study, HS-SPME combined with GC-MS/O was used to analyze the volatile flavor components of Strong aroma baijiu Daqu. DI-GC-O was used to choose the best extraction fiber to extract the representative overall aroma profile of Daqu. A total of 139 compounds were identified in the six different maturity stages of Daqu, and these compounds are of different types and concentrations. HS-SPME combined with GC-MS/O was used to analyze the aroma active substances in the finished Daqu, and a total of 43 aroma compounds were identified. The OAVs of 21 aromatic compounds were calculated based on the quantitative analysis results of MHS-SPME. Eighteen compounds with OAVs $\geq 1$ made significant contributions to the overall aroma of Daqu, including guaiacol, 4-ethyl-2-methoxy phenol, 2-ethyl-3,5-dimethylpyrazine, etc.
\end{abstract}

Keywords: baijiu; Daqu; MHS-SPME; DI-GC-O; volatile flavor components

\section{Introduction}

Baijiu, known as Chinese national liquor, is a part of Chinese traditional culture. As one of the daily alcoholic beverages, it is prevalent among Chinese people [1]. $Q u$, as the source of flavor and microorganism for baijiu, has received wide attention in baijiu production [2,3]. $Q u$ is a starter, saccharifying agent and aroma generator in baijiu production, and plays a vital role in baijiu making [1]. The main types of $Q u$ are Daqu, Xiaoqu, and Fuqu, and the primary raw materials of $Q u$ are wheat, sorghum, and bran, respectively. The relevant microbes that play a major role in the $Q u$ fermentation including moulds, yeasts, and bacteria [2]. There are 12 flavor types of baijiu, among which Strong, Light, Sauce, and Rice aroma baijiu are four basic flavor types, and other flavor types are derived from the basic flavors [4]. The 12 flavor types of baijiu are closely related to the kinds of $Q u$. According to the main technical characteristics of the 12 flavor types of baijiu and the primary production technology of $Q u$, the relationship between flavor types and $Q u$ is summarized as shown in Figure 1a.

Flavor wheel has been often used to help people understand and identify the flavor of a complicated product. The Baijiu flavor wheel [5] describes the sensory characteristics of baijiu from three aspects: aroma, taste, and mouthfeel (Figure S1). The aroma of raw materials, fermentation aroma, and aging aroma are three important aroma components for the Baijiu Flavor Wheel. $Q u$ aroma is one of the seven kinds of aroma to evaluate raw materials aroma. Daqu used in SAB is a kind of $Q u$. Crushed wheat and other raw materials are mixed with an appropriate amount of water to produce Daqu, which is then 
made into $Q u$-bricks with a specific mold. Then, the $Q u$-bricks were transferred to the fermentation room, appropriate straw use for isolation and moisture absorption. Pile the fermented $Q u$-bricks in the store room to be crushed for use. The immediate process of Daqu-making is demonstrated in Figure 1b. Daqu contains high protein and is rich in starch and other nutrients, which is the primary source of microorganisms [3]. It can provide microorganisms with hydrolytic enzymes that hydrolyze macromolecules in fermented grains. In the process, an aroma is produced, which we call $Q u$ aroma. Strong aroma baijiu (SAB) is one of the baijiu flavor types accounting for more than $70 \%$ of the market, for which sorghum, rice, sticky rice, wheat, and corn are used as the raw material. Its standard production technology is steamed grains mixed with Daqu are put into pit (jiaochi) for fermentation. After fermentation in the pit, solid-state distillation occurs with zeng (a kind of distillation device). Containers are used to collect and store baijiu. At present, the research on the flavor of SAB is aimed at the study of the overall flavor of baijiu [6], the change rule of flavor substances in the baijiu distillation process [7] and the characteristic aroma: roasted and mud-like aromas [8]. This study is carried out in view of the problem that the research on $Q u$ aroma is not sufficient.

a

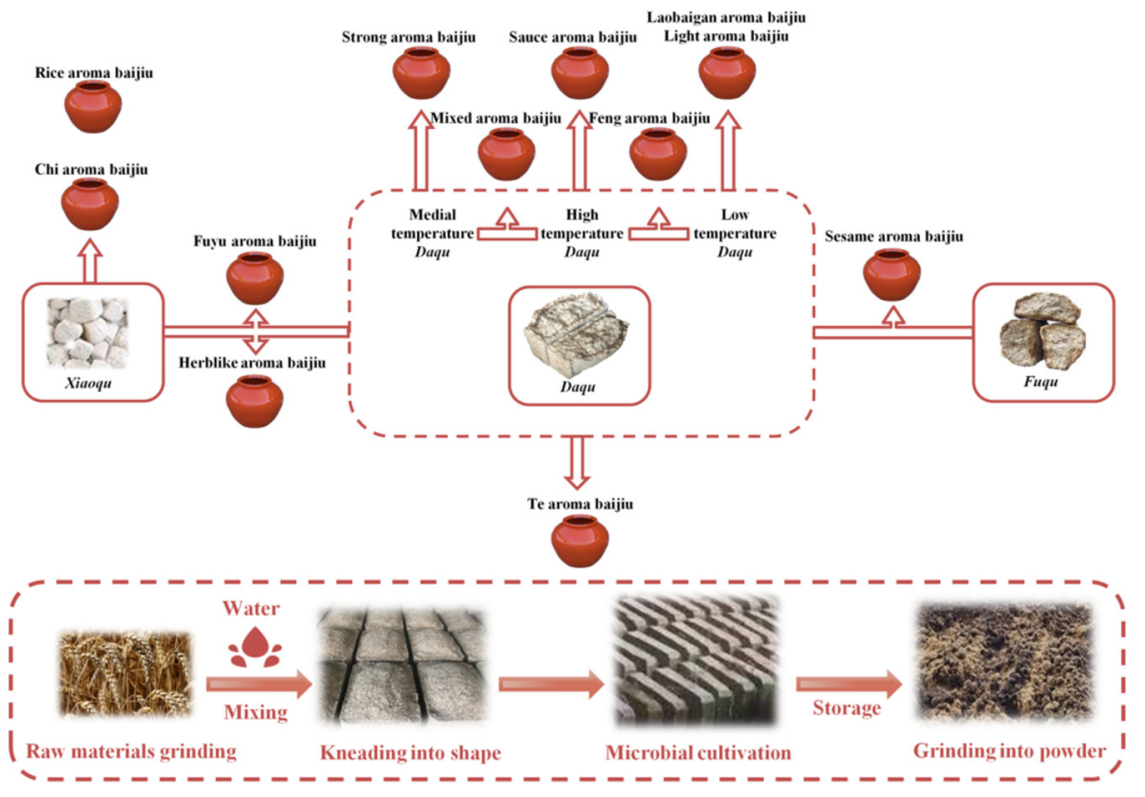

Figure 1. Relationship between 12 flavors of baijiu and the types of $Q u$ (a), the primary process of Daqu-making (b).

The samples in this experiment are six kinds of Daqu from the freshly pressed Daqu block in the production process to the Daqu before it is officially put into SAB production. At present, there are mainly two methods for the extraction of volatile compounds from solid samples: (1) To make the volatile compounds of the samples reach equilibrium in the headspace in the confined space, and then use the extraction fiber coated with unique materials to extract directly from the headspace gas of the samples; (2) To extract the volatile compounds from the solid to the liquid by using the principle of compound similarity compatibility. Then, the liquid sample was concentrated and extracted by the pretreatment method. At present, the pretreatment methods applied to the analysis of volatile components in solid samples are mainly solid phase microextraction (SPME), simultaneous distillation extraction (SDE), solvent assisted flavor evaporation (SAFE), etc. In this paper, HS-SPME was used to extract the aroma of Daqu.

Headspace solid phase microextraction (HS-SPME) is an incomplete method, which is easily affected by the matrix effect in the quantitative analysis of compounds in solid samples, which leads to inaccurate quantification. Multiple headspace solid phase microextraction (MHS-SPME) is a quantitative method developed based on HS-SPME. MHS-SPME 
has nothing to do with the distribution between the two phases, which can eliminate the matrix effect of the sample. At present, MHS-SPME has been successfully applied to the quantification of some volatile compounds in mushrooms [9,10], tomatoes [11], cheese [12], dry fermented sausage [13], wine [14,15] and alcoholic beverages [16]. This paper is the initial stage of the application of MHS-SPME to the analysis of volatiles in Daqu as we know it. Direct gas chromatography olfactometry (DI-GC-O) is a direct and effective technique to evaluate sensory flavor [17]. The extract was injected into a short unfilled capillary column directly connected to the GC sniffing port. Since there was no chromatographic separation, the method could evaluate the overall olfaction of the extract [18]. As shown in Figure 2, the volatile odors not separated by the chromatographic column (a) are smelled more quickly and directly than the column with packing (b). It has been used in the detection of orange juice [17], soy sauce [18], and baijiu samples [19,20] at present. Many researchers select extraction fiber according to the peak area of compounds. However, some studies showed that the aroma intensity detected by DI-GC-O correlated with the total peak area analyzed by GC-MS [18]. Therefore, DI-GC-O is listed as a good choice. The application of this method in solid samples such as Daqu has not been reported.

a

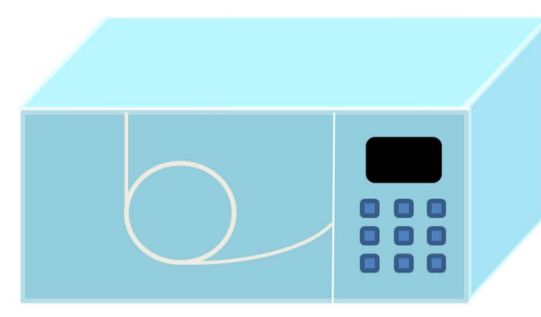

b

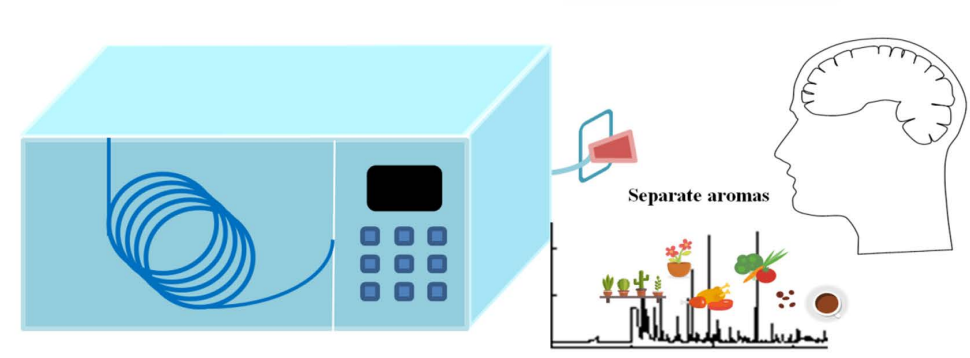

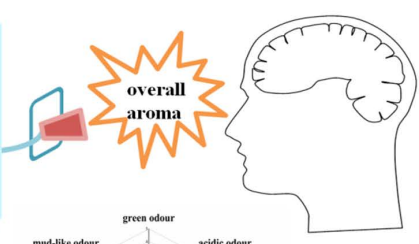
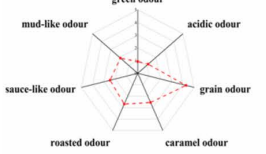
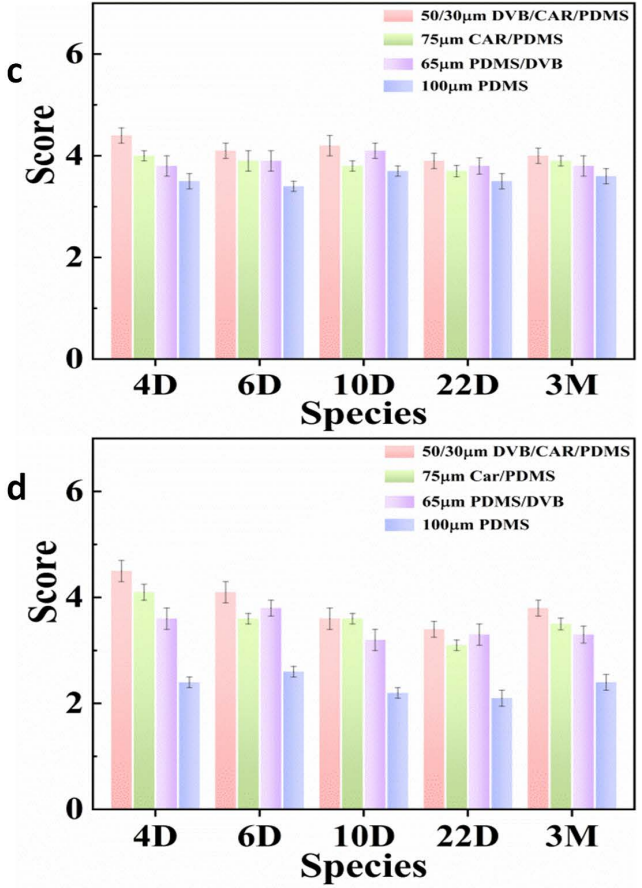

Figure 2. DI-GC-O sniffing diagram (a), with the short unfilled capillary column. GC-O sniffing diagram (b), with the packed column. Screening and optimization of extraction head by DI-GC-O Aroma similarity (c) and aroma intensity (d).

This research will introduce from the following aspects: (1) the extraction fiber was chosen with DI-GC-O; (2) analyses of aroma-active compounds from different maturity stages Daqu by HS-SPME combined with GC-MS/O; (3) key odor compounds of Daqu based on MHS-SPME quantitative analysis and aroma active value (OAV) calculation; and (4) it provides scientific support for the use of the stored Daqu in actual production in terms of its aroma richness.

\section{Materials and Methods}

\subsection{Chemical Standards, Reagents, and Materials}

2,6-dimethylpyrazine, nonanal, (E)-2-octenal, 2,3-dimethylpyrazine, 3-ethyl-2methylpyrazine, 2,3,5-trimethylpyrazine, 2-ethyl-3,5-dimethylpyrazine, benzaldehyde, (E)-2-nonanal, $\beta$-caryophyllene, $\gamma$-butyrolactone, phenylacetaldehyde, furfuryl alcohol, 2,4-decadienal, geranyl acetone, guaiacol, 4-ethyl-2-methoxyphenol, $\gamma$-nonanolactone, 2 - 
methoxy-4-vinylphenol, hexanal, 2-phenyl-2-butenal (all of them are chromatographic purity with at least 97\% purity, J\&K Scientific Ltd.). A C7-C30 n-alkane mixture was purchased from J\&K Scientific Ltd., Beijing, China. The internal standard 2-octanol (chromatographic purity with $98.0 \%$ purity), anhydrous ethanol (chromatography grade), and sodium chloride (analytical purity) were purchased from China National Pharmaceutical Group Corp. (Shanghai, China). High purity helium (99.999\%) was purchased from Beijing Yanglilai Chemical Gas Co., Ltd. (Beijing, China).

\subsection{Samples}

All were collected from the Gujing gongjiu Co., Ltd., Anhui, China. All samples were stored at $-20{ }^{\circ} \mathrm{C}$ separately in sealed plastic bags in the fridge until analysis.

There are several key time points for the fermentation process of the Daqu that we distinguish by the number of days. The samples for this experiment were collected for these key time points. The information of the sample is shown in Table S1.

\subsection{Sample Pretreatment}

The Daqu samples were smashed and screened over 40 mesh. Each different maturity stage of Daqu (as shown in Table S1) is mixed individually-sealed and stored in cold storage to ensure the reproducibility of sample extraction.

\subsection{Extraction of Volatile Components of Daqu}

\subsubsection{Selection of Extraction Fiber by DI-GC-O}

In this experiment, Daqu S-3M was used. The experimental tasters were composed of three highly trained tasters in our research group (All of them are members of the Key Laboratory of Brewing Molecular Engineering of China Light Industry who have received sensory training of baijiu evaluation).

In addition, $1.5 \mathrm{~g}$ Daqu sample was taken and placed in a cup at room temperature of $25^{\circ} \mathrm{C} \pm 1$ as reference. The overall odor intensity of Daqu S-3M was scored directly. The intensity of aroma was rated on 6 -point scale. $0=$ not smell, $1=$ very weak smell, $2=$ weak smell, 3 = moderate smell, 4 = strong smell, and 5 = very strong smell, and the overall flavor of samples was memorized.

Under the condition of different extraction fibers, the aroma components analyzed by DI-GC-O but not separated by a chromatography column were scored according to the flavor intensity of real Daqu. In the experiment process, the members of the sniffing group need to evaluate the samples to be tested in two aspects: (1) the overall flavor intensity of olfactory perception; (2) between the flavor of the extract and the original sample similarly. The reviewers need to sniff the actual sample again between the two sniffing experiments to enhance their impression. Each person sniffed three times and scored, averaging the results based on each person's score.

Gas chromatographic conditions: no-packed capillary column $(100 \mathrm{~cm} \times 25 \mathrm{~mm})$. The front inlet temperature was $250{ }^{\circ} \mathrm{C}$ in splitless mode. The extraction fiber was inserted into the injection port of a GC for $5 \mathrm{~min}$ to desorb, $\mathrm{N}_{2}(99.999 \%)$ as the carrier gas at $2.0 \mathrm{~mL} / \mathrm{min}$. The oven was $40{ }^{\circ} \mathrm{C}$ initially. Between the two injections, the column temperature was raised to $250{ }^{\circ} \mathrm{C}$ for $5 \mathrm{~min}$, and the column was aged to prevent cross contamination.

\subsubsection{Volatile Components Extracted by HS-SPME}

The extraction fiber was screened according to the previous experiment, the $50 / 30 \mu \mathrm{m}$ DVB/CAR/PDMS fiber was selected as extraction fiber, and $50^{\circ} \mathrm{C}$ as extraction temperature and $40 \mathrm{~min}$ as extraction time. In addition, $5 \mathrm{~g}$ of different maturity stages Daqu samples were weighed in a $20 \mathrm{~mL}$ headspace bottle, equilibrated at $50{ }^{\circ} \mathrm{C}$ for $20 \mathrm{~min}$ and extracted for $40 \mathrm{~min}$. Then, the extraction fiber was placed at $250{ }^{\circ} \mathrm{C}$ for $5 \mathrm{~min}$ resolution time in the GC injection port, analyzed with GC-MS subsequently.

The heating procedure, GC, and Mass spectrum conditions were using conditions in reference [21]. 


\subsection{Identification of Volatile Components}

\subsubsection{Volatiles Identification by GC-MS/O Analysis}

During the analysis, the evaluator must place the nose at the olfactory detector and record the retention time, aroma characteristics, and odor intensity during the olfactory process. Among them, the intensity range is $1-4$, and 1 means weak aroma intensity; 2 means the aroma intensity is slightly stronger; 3 means obvious aroma intensity; 4 means strong aroma intensity. Each person sniffed three times to ensure the accuracy of the results.

GC conditions: DB-WAX column $(60 \mathrm{~m} \times 0.25 \mathrm{~mm} \times 0.25 \mu \mathrm{m})$ and HP-5 column $(30 \mathrm{~m} \times 0.25 \mathrm{~mm} \times 0.25 \mu \mathrm{m})$; Helium $(99.999 \%)$ was used as a carrier gas at a constant flow rate of $1.0 \mathrm{~mL} / \mathrm{min}$. The temperature of the injection port was $250^{\circ} \mathrm{C}$, and the resolution time was $5 \mathrm{~min}$.

The heating procedure was the same as the Section 2.4.2 heating procedure.

Olfactory detector: With the carrier gas, the effluent from the column is divided into the mass spectrometer detector and the olfactory detector in 1:1 mode. The olfactory temperature and the transmission line temperature of the olfactory end are $250{ }^{\circ} \mathrm{C}$. To prevent the nose from drying out after a long time of sniffing, the olfactory temperature and nitrogen line are passed through deionized water.

\subsubsection{Qualitative Analysis}

NIST 11 library search, standard comparison, and retention index were used for qualitative analysis.

NIST 11 library search: gas chromatography-mass spectrometry data are analyzed and processed by Chemstation software (1989-2010 Agilent Technologies, Inc., Santa Clara, CA, USA). Firstly, the background of the spectrum was deducted, and then the library search was carried out. The qualitative results of the library were required to have a similarity of more than $80 \%$.

Standard comparison: under the same chromatographic conditions, the sample and standard can be analyzed. If the retention time and mass spectrum of the corresponding peaks of the two chromatograms are the same, they can be identified as the same compound.

Retention index (RI) qualitative was calculated [6] using a C7 C30 n-alkane mixture under the same GC-MS conditions as the samples.

\subsection{Quantitative and $O A V$ Analysis}

MHS-SPME is used as a quantitative method. The appropriate sample volume is the critical factor in obtaining the exponential decrease of peak area of each analyte in the continuous extraction process. In this experiment, the previous experimental methods and results [21] were used, for which $35 \mathrm{mg}$ samples were selected for analysis. Weigh $35 \mathrm{mg}$ of Daqu sample into a $20 \mathrm{~mL}$ headspace bottle, and carry out the experimental operation according to the HS-SPME method described before. The interval between two adjacent extractions was $20 \mathrm{~min}$.

Quantitative: calibration with an external standard curve, take $100 \mu \mathrm{L}$ diluted the mixed standard solution in the headspace bottle, and then analyze by MHS-SPME under the same conditions as the sample. All analyses were repeated in triplicate.

The OAV value was calculated based on the ratio of the target odorant concentration to its threshold.

\subsection{Statistical Analysis}

The compounds were analyzed by Chemical Station (Agilent, Santa Clara, CA, USA). Origin Pro 2021 was used for data analysis and related graphics drawing. SIMCA-14.1 was used to analyze PCA (Umetrics, Umea, Sweden). 


\section{Results}

\subsection{Screening and Optimization of Extraction Fiber by DI-GC-O}

This method injected extraction fiber with an adsorbed compound into an empty chromatographic column connected with the GC injection port and olfactory detector. Then, the sensory evaluator directly smelled the aroma extract which was not separated by the chromatographic column. The best extraction conditions determined by the overall flavor similarity combined with sensory evaluation between the extract and the real sample were investigated. This method avoids the separation effect of the chromatographic column and restores the authenticity of the sample to the greatest extent. DI-GC-O was used to evaluate the extraction effect of four different extraction fibers in Daqu.

The order of overall aroma intensity and similarity of different extraction fibers is 50/30 $\mu \mathrm{m}$ DVB/CAR/PDMS > $75 \mu \mathrm{m}$ CAR/PDMS > $65 \mu \mathrm{m}$ PDMS/DVB > $100 \mu \mathrm{m}$ PDMS, which can be seen from Figure $2 c$,d. It shows that the 50/30 $\mu \mathrm{m}$ DVB/CAR/PDMS extraction fiber has certain advantages in the extraction of aroma compounds in Daqu, which can extract more compounds and most likely reflect the overall aroma of Daqu.

\subsection{Analysis of Daqu at Different Maturity Stages by HS-SPME Combined with GC-MS}

According to the HS-SPME extraction method in Section 2.4.2, 139 compounds were identified in six different maturity stages Daqu samples (shown in Table 1), including 36 esters, 7 acids and ketones, 22 alcohols, 4 lactones, 8 phenols and furans, 14 aldehydes, 12 nitrogenous compounds, 9 terpenes, 3 sulfur compounds and 9 others. The number of volatile compounds in six different mature stages Daqu was in the order of "S-3M > S-22D $>$ S-10D $=$ S-0D > S-6D > S-4D". The variation of compounds types in Daqu is shown in Figure $3 \mathrm{~A}$, and it can be found that the aroma of Daqu S-3M was more abundant.

Table 1. Analysis of volatile components in Daqu at different maturity stages.

\begin{tabular}{|c|c|c|c|c|c|c|c|c|c|c|}
\hline \multirow{2}{*}{ No. } & \multirow{2}{*}{ Compound } & \multirow{2}{*}{$\begin{array}{c}\text { CAS } \\
\text { Number }\end{array}$} & \multirow{2}{*}{ RI } & \multirow{2}{*}{ Identification ${ }^{\text {a }}$} & \multicolumn{6}{|c|}{ Area Percentage $\%$ b } \\
\hline & & & & & S-0D & S-4D & S-6D & S-10D & S-22D & S-3M \\
\hline \multicolumn{11}{|l|}{ Esters } \\
\hline 1 & $\begin{array}{c}\text { Ethyl } \\
\text { 3-methylbutanoate }\end{array}$ & $108-64-5$ & 1074 & MS,RI & - & 0.525 & 0.765 & - & - & - \\
\hline 2 & Isoamyl acetate & $123-92-2$ & 1123 & S,MS,RI & - & 1.126 & 0.159 & - & - & 0.082 \\
\hline 3 & Ethyl pentanoate & $539-82-2$ & 1135 & S,MS,RI & - & - & - & - & 0.335 & - \\
\hline 4 & Ethyl hexanoate & $123-66-0$ & 1233 & S,MS,RI & 3.760 & 2.489 & 1.230 & 8.698 & 8.656 & 1.122 \\
\hline 5 & Ethyl heptanoate & $106-30-9$ & 1334 & MS,RI & - & 0.367 & 0.330 & 0.485 & 0.505 & 0.270 \\
\hline 6 & Ethyl lactate & $97-64-3$ & 1344 & S,MS,RI & - & 0.800 & 0.330 & - & - & 0.530 \\
\hline 7 & Hexyl formate & $629-33-4$ & 1350 & S,MS,RI & 2.136 & 0.159 & 0.137 & 0.869 & 0.358 & 0.562 \\
\hline 8 & $\begin{array}{l}\text { 1-Methylheptyl } \\
\text { acetate }\end{array}$ & $2051-50-5$ & 1363 & MS & - & - & - & - & 0.038 & - \\
\hline 9 & Ethyl octanoate & $106-32-1$ & 1435 & S,MS,RI & 1.514 & 1.263 & 1.177 & 0.518 & 0.796 & 0.404 \\
\hline 10 & Ethyl nonanoate & $123-29-5$ & 1537 & S,MS,RI & - & 0.159 & 0.179 & 0.587 & 0.345 & 0.237 \\
\hline 11 & $\begin{array}{l}\text { 2-Hydroxyethyl } \\
\text { hexanoate }\end{array}$ & $6946-90-3$ & 1545 & MS,RI & - & 1.789 & - & - & - & - \\
\hline 12 & $\begin{array}{l}\text { 3-(Methylthio)- } \\
\text { propanoic acid ethyl } \\
\text { ester }\end{array}$ & $13327-56-5$ & 1572 & MS,RI & - & 0.665 & 0.268 & 0.111 & 0.132 & 0.137 \\
\hline 13 & Hexyl hexanoate & $6378-65-0$ & 1611 & S,MS,RI & - & - & - & - & - & 0.066 \\
\hline 14 & Ethyl 2-furoate & $614-99-3$ & 1631 & MS,RI & 0.230 & - & - & - & - & - \\
\hline 15 & Ethyl decanoate & $110-38-3$ & 1640 & S,MS,RI & - & 1.056 & 1.231 & 0.292 & 0.343 & 0.223 \\
\hline 16 & Ethyl benzoate & $93-89-0$ & 1674 & S,MS,RI & 0.365 & 0.380 & 0.331 & 0.289 & 0.150 & 0.129 \\
\hline 17 & Diethyl succinate & $123-25-1$ & 1681 & S,MS,RI & 0.118 & 3.024 & 1.132 & 0.075 & 0.490 & 0.582 \\
\hline 18 & Methyl salicylate & $119-36-8$ & 1786 & S,MS,RI & 0.290 & 0.249 & 0.114 & 0.382 & 0.482 & 0.371 \\
\hline 19 & Ethyl phenylacetate & $101-97-3$ & 1793 & S,MS,RI & 0.199 & 3.155 & 0.445 & 1.034 & 0.765 & 0.815 \\
\hline 20 & Methyl dodecanoate & $111-82-0$ & 1803 & S,MS,RI & 0.084 & 0.017 & 0.026 & 0.088 & 0.066 & 0.280 \\
\hline 21 & $\begin{array}{c}\text { Ethyl } \\
\text { 2-hydroxybenzoate }\end{array}$ & $118-61-6$ & 1820 & S,MS,RI & - & - & - & - & 0.069 & 0.198 \\
\hline 22 & Phenethyl acetate & $103-45-7$ & 1824 & S,MS,RI & - & 0.559 & 0.080 & 0.154 & - & - \\
\hline 23 & Ethyl nicotinate & $614-18-6$ & 1825 & S,MS,RI & 0.063 & - & 0.110 & 0.136 & 0.119 & - \\
\hline 24 & Ethyl dodecanoate & $106-33-2$ & 1843 & S,MS,RI & - & 0.689 & 0.889 & 0.562 & 0.596 & 0.480 \\
\hline
\end{tabular}


Table 1. Cont.

\begin{tabular}{|c|c|c|c|c|c|c|c|c|c|c|}
\hline \multirow{2}{*}{ No. } & \multirow{2}{*}{ Compound } & \multirow{2}{*}{$\begin{array}{c}\text { CAS } \\
\text { Number }\end{array}$} & \multirow{2}{*}{ RI } & \multirow{2}{*}{ Identification $^{\mathrm{a}}$} & \multicolumn{6}{|c|}{ Area Percentage $\%$ b } \\
\hline & & & & & S-0D & S-4D & S-6D & S-10D & S-22D & S-3M \\
\hline \multicolumn{11}{|l|}{ Esters } \\
\hline 25 & $\begin{array}{c}\text { Ethyl } \\
\text { 3-phenylpropanoate }\end{array}$ & $2021-28-5$ & 1884 & S,MS,RI & 0.125 & 0.103 & 0.082 & 0.033 & 0.113 & 0.052 \\
\hline 26 & $\begin{array}{l}\text { Phenylethyl } \\
\text { isovalerate }\end{array}$ & $140-26-1$ & 1978 & S,MS & - & 0.025 & 0.021 & - & - & 0.015 \\
\hline 27 & Ethyl tetradecanoate & $124-06-1$ & 2022 & S,MS,RI & 0.182 & 2.003 & 2.891 & 2.712 & 3.127 & 0.100 \\
\hline 28 & Ethyl cinnamate & $103-36-6$ & 2094 & S,MS,RI & - & - & - & - & - & 0.042 \\
\hline 29 & Ethyl pentadecanoate & $41114-00-5$ & 2099 & S,MS,RI & - & 0.494 & 0.715 & 1.320 & 1.186 & 1.251 \\
\hline 30 & Methyl hexadecanoate & 112-39-0 & 2181 & MS & - & - & - & - & - & 0.118 \\
\hline 31 & Ethyl hexadecanoate & $628-97-7$ & 2227 & S,MS,RI & 2.777 & 14.552 & 18.454 & 23.904 & 22.731 & 29.140 \\
\hline 32 & Ethyl 9-hexadecenoate & $54546-22-4$ & 2259 & S,MS,RI & - & 1.298 & 1.589 & 0.882 & 1.226 & 0.997 \\
\hline 33 & Ethyl heptadecanoate & $14010-23-2$ & 2350 & S,MS,RI & - & 0.056 & 0.067 & 0.114 & - & 0.117 \\
\hline 34 & Ethyl octadecanoate & $111-61-5$ & 2483 & S,MS,RI & - & 0.318 & 0.364 & 0.602 & 0.564 & 0.648 \\
\hline 35 & Ethyl Oleate & $111-62-6$ & 2475 & S,MS,RI & 0.457 & 2.843 & 3.961 & 6.784 & 6.399 & 7.778 \\
\hline \multirow[t]{2}{*}{36} & $\begin{array}{c}\text { Methyl } \\
\text { octadecadienoate }\end{array}$ & $112-63-0$ & 2588 & S,MS & 0.107 & 3.214 & 3.614 & 5.067 & 6.111 & 6.372 \\
\hline & Subtotal & & & & 12.410 & 43.381 & 40.692 & 55.695 & 55.703 & 53.120 \\
\hline \multicolumn{11}{|l|}{ Acids } \\
\hline 37 & Acetic acid & $64-19-7$ & 1458 & S,MS,RI & 0.756 & 0.487 & 0.562 & 0.674 & 0.851 & 0.729 \\
\hline 38 & $\begin{array}{l}\text { 2-Methylpropionic } \\
\text { acid }\end{array}$ & $79-31-2$ & 1569 & MS,RI & - & 0.645 & 0.214 & 0.077 & 0.077 & 0.085 \\
\hline 39 & 3-Methylbutanoic acid & $503-74-2$ & 1672 & MS,RI & 0.251 & 9.765 & 3.172 & 1.224 & 1.596 & 1.514 \\
\hline 40 & Hexanoic acid & $142-62-1$ & 1846 & S,MS,RI & 1.253 & 0.165 & 0.037 & 0.288 & 0.185 & 0.336 \\
\hline 41 & Heptanoic acid & $111-14-8$ & 1940 & MS,RI & 0.101 & 0.024 & 0.036 & - & - & - \\
\hline 42 & Octanoic acid & $124-07-2$ & 2031 & S,MS,RI & 0.241 & - & - & - & 0.081 & 0.053 \\
\hline \multirow{2}{*}{43} & Decanoic acid & $334-48-5$ & 2247 & MS,RI & 0.357 & - & - & - & - & - \\
\hline & Subtotal & & & & 2.959 & 11.086 & 4.021 & 2.263 & 2.789 & 2.717 \\
\hline \multicolumn{11}{|c|}{ Alcohols } \\
\hline 44 & 3-Methyl-1-butanol & $123-51-3$ & 1202 & MS,RI & 0.373 & 4.540 & 4.454 & 2.077 & 0.514 & 4.323 \\
\hline 45 & 1-Pentanol & $71-41-0$ & 1246 & MS & 0.827 & - & - & - & - & 0.873 \\
\hline 46 & 3-Methyl-2-buten-1-ol & $556-82-1$ & 1321 & S,MS,RI & - & - & - & 0.089 & 0.020 & 0.342 \\
\hline 47 & 3-Octanol & $589-98-0$ & 1391 & MS,RI & 0.328 & - & - & - & 0.415 & - \\
\hline 48 & 1-Octen-3-ol & $3391-86-4$ & 1448 & S,MS,RI & 0.854 & 0.484 & 0.826 & 0.271 & 0.237 & 0.311 \\
\hline 49 & 1-Heptanol & $111-70-6$ & 1452 & S,MS,RI & 1.342 & 0.055 & 0.126 & 0.671 & 0.497 & 0.151 \\
\hline 50 & $\begin{array}{l}\text { 6-Methyl-5-hepten-2- } \\
\text { ol }\end{array}$ & $1569-60-4$ & 1462 & MS,RI & 0.489 & - & - & - & - & - \\
\hline 51 & 2-Ethylhexanol & $104-76-7$ & 1488 & S,MS,RI & 0.874 & 0.040 & 0.057 & 0.545 & 0.193 & 0.413 \\
\hline 52 & 2-Nonanol & $628-99-9$ & 1517 & S,MS,RI & - & 0.083 & 0.101 & 0.180 & 0.095 & 0.043 \\
\hline 53 & 1-Octanol & $111-87-5$ & 1556 & S,MS,RI & 0.428 & 0.053 & 0.066 & 0.148 & 0.097 & 0.139 \\
\hline 54 & 2,3-Butanediol & $513-85-9$ & 1577 & S,MS,RI & 1.364 & 0.056 & 0.083 & 0.646 & 0.403 & 0.699 \\
\hline 55 & (E)-2-Octenol & 18409-17-1 & 1616 & S,MS,RI & 0.224 & 0.081 & 0.112 & - & - & - \\
\hline 56 & 1-Nonanol & 143-08-8 & 1659 & S,MS,RI & 0.166 & 0.055 & 0.051 & 0.054 & 0.056 & 0.061 \\
\hline 57 & (Z)-3-Nonen-1-ol & $10340-23-5$ & 1684 & MS,RI & 0.104 & - & - & - & - & - \\
\hline 58 & $\alpha$-Terpineol & $98-55-5$ & 1699 & S,MS,RI & 0.747 & 0.038 & 0.039 & 0.042 & 0.281 & 0.038 \\
\hline 59 & Methionol & $505-10-2$ & 1721 & S,MS,RI & - & 0.071 & 0.118 & 0.108 & 0.046 & 0.047 \\
\hline 60 & 2-Phenyl-2-propanol & $617-94-7$ & 1762 & S,MS,RI & - & - & - & 0.117 & 0.037 & 0.097 \\
\hline 61 & 1-Phenylethanol & $98-85-1$ & 1819 & S,MS,RI & - & - & - & - & 0.057 & 0.019 \\
\hline 62 & Benzyl alcohol & $100-51-6$ & 1878 & S,MS,RI & 0.188 & 0.228 & 0.176 & 1.318 & 1.033 & 2.233 \\
\hline 63 & Phenylethyl Alcohol & $60-12-8$ & 1907 & S,MS,RI & 3.652 & 14.526 & 11.272 & 7.081 & 14.432 & 11.298 \\
\hline 64 & 2-Phenyl-1-propanol & $1123-85-9$ & 1923 & S,MS,RI & - & - & - & 0.052 & 0.070 & 0.047 \\
\hline \multirow[t]{2}{*}{65} & 2-Phenyl-1-butanol & 2035-94-1 & 1973 & MS & - & 0.025 & 0.041 & - & - & - \\
\hline & Subtotal & & & & 11.960 & 20.335 & 17.523 & 13.400 & 18.482 & 21.134 \\
\hline \multicolumn{11}{|l|}{ lactones } \\
\hline 66 & $\gamma$-Butyrolactone & $96-48-0$ & 1643 & S,MS,RI & 0.130 & 0.029 & 0.136 & 0.195 & 0.164 & 0.113 \\
\hline 67 & $\begin{array}{l}\text { 5-Butyldihydro-2(3H)- } \\
\text { furanone }\end{array}$ & $104-50-7$ & 1917 & S,MS,RI & 0.133 & 0.156 & 0.179 & - & - & 0.106 \\
\hline 68 & $\gamma$-Nonanolactone & $104-61-0$ & 2017 & S,MS,RI & 1.445 & 0.389 & 0.237 & 0.142 & 0.142 & 0.162 \\
\hline \multirow[t]{2}{*}{69} & $\begin{array}{l}\text { (Z)-6-Dodeceno- } \gamma \text { - } \\
\text { lactone }\end{array}$ & $18679-18-0$ & 2438 & S,MS & - & 0.076 & - & - & - & - \\
\hline & Subtotal & & & & 1.708 & 0.650 & 0.552 & 0.337 & 0.307 & 0.382 \\
\hline Phenols & & & & & & & & & & \\
\hline 70 & Guaiacol & $90-05-1$ & 1865 & S,MS,RI & 0.177 & - & - & 1.150 & 0.136 & 0.172 \\
\hline 71 & Creosol & $93-51-6$ & 1952 & S,MS,RI & - & 0.432 & 0.029 & 0.101 & 0.078 & 0.073 \\
\hline 72 & $o$-Cresol & $95-48-7$ & 1990 & S,MS & - & - & - & - & - & 0.029 \\
\hline
\end{tabular}


Table 1. Cont.

\begin{tabular}{|c|c|c|c|c|c|c|c|c|c|c|}
\hline \multirow{2}{*}{ No. } & \multirow{2}{*}{ Compound } & \multirow{2}{*}{$\begin{array}{l}\text { CAS } \\
\text { Number }\end{array}$} & \multirow{2}{*}{ RI } & \multirow{2}{*}{ Identification $^{a}$} & \multicolumn{6}{|c|}{ Area Percentage $\%$ b } \\
\hline & & & & & S-OD & S-4D & S-6D & S-10D & S-22D & S-3M \\
\hline \multicolumn{11}{|c|}{ Phenols } \\
\hline 73 & Phenol & $108-95-2$ & 1993 & S,MS,RI & 0.400 & 0.041 & 0.054 & 0.874 & 0.285 & 0.373 \\
\hline 74 & $\begin{array}{l}\text { 4-Ethyl-2- } \\
\text { methoxyphenol }\end{array}$ & $2785-89-9$ & 2015 & S,MS,RI & 3.002 & 13.076 & 17.783 & 0.555 & 0.943 & 0.490 \\
\hline 75 & 4-Ethylphenol & $123-07-9$ & 2142 & S,MS,RI & 0.141 & 0.091 & 0.112 & 0.071 & 0.034 & 0.137 \\
\hline 76 & $\begin{array}{l}\text { 2-Methoxy-4- } \\
\text { vinylphenol }\end{array}$ & $7786-61-0$ & 2177 & $\mathrm{~S}, \mathrm{MS}, \mathrm{RI}$ & 0.646 & 3.237 & 8.710 & 0.969 & 0.696 & 0.319 \\
\hline \multirow[t]{2}{*}{77} & $\begin{array}{c}\text { 2,4-Bis(1,1- } \\
\text { dimethylethyl)- } \\
\text { phenol }\end{array}$ & $96-76-4$ & 2296 & S,MS,RI & 10.538 & 0.135 & 0.291 & 1.228 & 0.611 & 0.962 \\
\hline & Subtotal & & & & 14.903 & 17.012 & 26.978 & 4.949 & 2.785 & 2.554 \\
\hline \multicolumn{11}{|c|}{ Ketones } \\
\hline 78 & 3-Octanone & $106-68-3$ & 1254 & S,MS,RI & - & 0.114 & 0.194 & - & - & - \\
\hline 79 & 2-Octanone & $111-13-7$ & 1286 & S,MS,RI & 5.823 & 4.586 & 4.793 & 7.414 & 8.267 & 2.411 \\
\hline 80 & $\begin{array}{l}\text { 6-Methyl-5-hepten-2- } \\
\text { one }\end{array}$ & $110-93-0$ & 1340 & $\mathrm{~S}, \mathrm{MS}, \mathrm{RI}$ & 0.296 & - & - & - & - & 0.316 \\
\hline 81 & 2-Nonanone & $821-55-6$ & 1390 & S,MS,RI & - & - & - & 0.143 & - & 0.214 \\
\hline 82 & 3,5-Octadien-2-one & $38284-27-4$ & 1525 & MS,RI & 0.083 & - & - & - & - & 0.030 \\
\hline 83 & Acetophenone & $98-86-2$ & 1661 & S,MS,RI & 0.701 & 0.082 & 0.104 & 0.594 & 0.521 & 0.871 \\
\hline \multirow{2}{*}{84} & Butyrophenone & $495-40-9$ & 1805 & S,MS,RI & - & - & - & - & - & 0.158 \\
\hline & Subtotal & & & & 6.903 & 4.782 & 5.090 & 8.151 & 8.788 & 4.000 \\
\hline \multicolumn{11}{|c|}{$\begin{array}{l}\text { Nitrogenous } \\
\text { compounds }\end{array}$} \\
\hline 85 & 2-Methylpyrazine & $109-08-0$ & 1268 & S,MS,RI & 0.170 & - & - & 0.274 & 0.298 & 0.321 \\
\hline 86 & 2,5-Dimethylpyrazine & $123-32-0$ & 1324 & S,MS,RI & - & - & - & 0.054 & 0.157 & 0.186 \\
\hline 87 & 2,6-Dimethylpyrazine & $108-50-9$ & 1330 & S,MS,RI & 0.216 & - & - & 0.374 & 0.330 & 0.626 \\
\hline 88 & 2,3-Dimethylpyrazine & $5910-89-4$ & 1349 & S,MS,RI & - & - & - & 0.262 & 0.128 & 0.186 \\
\hline 89 & $\begin{array}{l}\text { 2-Ethyl-6- } \\
\text { methylpyrazine }\end{array}$ & $13925-03-6$ & 1386 & S,MS,RI & - & - & - & 0.399 & 0.244 & 0.411 \\
\hline 90 & $\begin{array}{c}\text { 2,3,5- } \\
\text { Trimethylpyrazine }\end{array}$ & $14667-55-1$ & 1405 & S,MS,RI & 0.097 & 0.029 & 0.070 & 1.440 & 0.905 & 1.542 \\
\hline 91 & $\begin{array}{c}\text { 2-Ethyl-3,5- } \\
\text { dimethylpyrazine }\end{array}$ & $13925-07-0$ & 1463 & S,MS,RI & - & - & - & 0.663 & 0.203 & 0.617 \\
\hline 92 & Tetramethylpyrazine & $1124-11-4$ & 1476 & S,MS,RI & 0.262 & - & - & 1.710 & 0.544 & 2.068 \\
\hline 93 & $\begin{array}{l}\text { 2-Ethenyl-6- } \\
\text { methylpyrazine }\end{array}$ & $13925-09-2$ & 1494 & MS,RI & - & - & - & 0.403 & 0.490 & 0.533 \\
\hline 94 & $\begin{array}{c}\text { 2-Ethyl-3,5,6- } \\
\text { trimethylpyrazine }\end{array}$ & $17398-16-2$ & 1514 & MS,RI & - & - & - & 0.122 & 0.073 & 0.116 \\
\hline 95 & 2-Acetylpyrrole & $1072-83-9$ & 1966 & S,MS,RI & 0.138 & - & - & 0.030 & 0.086 & 0.139 \\
\hline \multirow[t]{2}{*}{96} & $\begin{array}{l}\text { 1H-Pyrrole-2- } \\
\text { carboxaldehyde }\end{array}$ & $1003-29-8$ & 2013 & $\mathrm{~S}, \mathrm{MS}, \mathrm{RI}$ & - & - & - & - & - & 0.482 \\
\hline & Subtotal & & & & 0.884 & 0.029 & 0.070 & 5.729 & 3.457 & 7.228 \\
\hline \multicolumn{11}{|c|}{ Aldehydes } \\
\hline 97 & Hexanal & $66-25-1$ & 1084 & MS,RI & 1.310 & - & - & 0.744 & 0.631 & 0.819 \\
\hline 98 & (E)-2-Heptenal & $18829-55-5$ & 1325 & MS,RI & 0.824 & - & - & - & - & - \\
\hline 99 & Nonanal & $124-19-6$ & 1396 & S,MS,RI & 0.628 & 0.034 & 0.264 & 0.206 & 1.042 & 0.245 \\
\hline 100 & (E)-2-Octenal & $2548-87-0$ & 1431 & S,MS,RI & 0.282 & - & - & - & - & 0.055 \\
\hline 101 & 3-Furaldehyde & $498-60-2$ & 1474 & S,MS,RI & - & - & - & - & 0.095 & 0.118 \\
\hline 102 & Decanal & $112-31-2$ & 1501 & MS,RI & 1.140 & - & - & - & - & 0.143 \\
\hline 103 & Benzaldehyde & $100-52-7$ & 1533 & S,MS,RI & 1.025 & 0.470 & 0.215 & 2.004 & 1.259 & 2.053 \\
\hline 104 & (E)-2-Nonenal & $18829-56-6$ & 1539 & S,MS,RI & 0.683 & - & 0.082 & - & - & 0.158 \\
\hline 105 & Phenylacetaldehyde & $122-78-1$ & 1654 & S,MS,RI & 0.412 & 0.524 & 0.367 & 0.661 & 0.734 & 0.636 \\
\hline 106 & $(E, E)-2,4$-Nonadienal & $5910-87-2$ & 1709 & S,MS,RI & 0.229 & - & - & - & - & - \\
\hline 107 & 3-Ethylbenzaldehyde & $34246-54-3$ & 1718 & S,MS & 0.059 & 0.012 & 0.033 & 0.023 & 0.021 & 0.024 \\
\hline 108 & $(E, E)$-2,4-Decadienal & $25152-84-5$ & 1818 & S,MS,RI & 5.750 & 0.054 & 0.080 & 0.290 & - & 0.147 \\
\hline 109 & $\begin{array}{l}\text { 5-Methyl-2- } \\
\text { thiophenecarboxaldehyde }\end{array}$ & $13679-70-4$ & 1825 & MS & - & - & - & 0.054 & 0.016 & 0.011 \\
\hline 110 & 2-Phenyl-2-butenal & 4411-89-6 & 1931 & S,MS,RI & 0.037 & 0.155 & 0.413 & 0.179 & 0.369 & 0.457 \\
\hline & Subtotal & & & & 12.381 & 1.249 & 1.455 & 4.162 & 4.169 & 4.866 \\
\hline \multicolumn{11}{|c|}{ Furans } \\
\hline 111 & 2-Pentylfuran & $3777-69-3$ & 1229 & S,MS,RI & - & - & - & - & - & 0.060 \\
\hline 112 & 5-Methyl furfural & $620-02-0$ & 1584 & S,MS,RI & - & - & - & - & 0.125 & 0.229 \\
\hline 113 & $\begin{array}{l}\text { 2-Acetyl-5- } \\
\text { methylfuran }\end{array}$ & $1193-79-9$ & 1625 & S,MS,RI & 0.087 & - & - & - & - & 0.038 \\
\hline 114 & Furfuryl alcohol & 98-00-0 & 1667 & S,MS,RI & - & 0.262 & 0.308 & 0.431 & 0.177 & 0.546 \\
\hline
\end{tabular}


Table 1. Cont.

\begin{tabular}{|c|c|c|c|c|c|c|c|c|c|c|}
\hline \multirow{2}{*}{ No. } & \multirow{2}{*}{ Compound } & \multirow{2}{*}{$\begin{array}{c}\text { CAS } \\
\text { Number }\end{array}$} & \multirow{2}{*}{ RI } & \multirow{2}{*}{ Identification $^{a}$} & \multicolumn{6}{|c|}{ Area Percentage $\%{ }^{b}$} \\
\hline & & & & & S-0D & S-4D & S-6D & S-10D & S-22D & S-3M \\
\hline \multicolumn{11}{|c|}{ Furans } \\
\hline 115 & $\begin{array}{l}\text { 5-Methyl-2- } \\
\text { furanmethanol }\end{array}$ & $3857-25-8$ & 1726 & MS,RI & - & - & - & - & 0.100 & 0.108 \\
\hline 116 & $\begin{array}{l}\text { 3-Methyl-2(5H)- } \\
\text { furanone }\end{array}$ & 22122-36-7 & 1732 & S,MS,RI & - & - & - & - & 0.170 & 0.200 \\
\hline 117 & 3-Phenylfuran & $13679-41-9$ & 1861 & MS,RI & - & - & 0.028 & 0.090 & 0.066 & 0.031 \\
\hline \multirow[t]{2}{*}{118} & Dibenzofuran & $132-64-9$ & 2277 & S,MS,RI & 3.286 & 0.531 & 0.794 & 1.425 & 0.661 & 0.710 \\
\hline & Subtotal & & & & 3.373 & 0.793 & 1.130 & 1.946 & 1.299 & 1.922 \\
\hline \multicolumn{11}{|c|}{ Terpenes } \\
\hline 119 & Limonene & $138-86-3$ & 1180 & MS,RI & 0.504 & - & - & - & - & - \\
\hline 120 & $\alpha$-Copaene & $3856-25-5$ & 1490 & MS,RI & 0.398 & - & - & - & - & - \\
\hline 121 & Linanool & $78-70-6$ & 1547 & MS,RI & 1.215 & - & - & - & - & - \\
\hline 122 & $\beta$-Caryophyllene & $87-44-5$ & 1598 & S,MS,RI & 17.526 & 0.049 & 0.103 & 0.996 & 0.908 & 0.237 \\
\hline 123 & $\begin{array}{c}\text { 2-Isopropyl-5- } \\
\text { methylcyclohexanol }\end{array}$ & 1490-04-6 & 1640 & MS,RI & 0.299 & - & - & - & - & - \\
\hline 124 & $(E)$ - $\beta$-farnesene & $18794-84-8$ & 1667 & MS,RI & 0.746 & - & - & - & - & - \\
\hline 125 & Humulene & $6753-98-6$ & 1671 & MS,RI & 0.150 & - & - & - & - & - \\
\hline 126 & (S)- $\beta$-bisabolene & $495-61-4$ & 1727 & MS,RI & 0.230 & - & 0.009 & - & - & - \\
\hline \multirow[t]{2}{*}{127} & Geranyl acetone & $3796-70-1$ & 1856 & S,MS,RI & 6.331 & 0.044 & 0.074 & 0.467 & 0.050 & 0.071 \\
\hline & Subtotal & & & & 27.399 & 0.092 & 0.186 & 1.463 & 0.957 & 0.308 \\
\hline \multicolumn{11}{|c|}{$\begin{array}{c}\text { Sulfur } \\
\text { compounds }\end{array}$} \\
\hline 128 & Benzothiazole & $95-16-9$ & 1955 & S,MS,RI & 0.268 & 0.049 & 0.017 & 0.025 & 0.039 & 0.046 \\
\hline 129 & 4,5-Dimethylthiazole & $3581-91-7$ & 1377 & MS,RI & 0.162 & - & - & - & - & 0.198 \\
\hline \multirow{2}{*}{130} & Dimethyl trisulfide & $3658-80-8$ & 1384 & S,MS,RI & - & - & - & - & 0.034 & - \\
\hline & Subtotal & & & & 0.430 & 0.049 & 0.017 & 0.025 & 0.073 & 0.244 \\
\hline \multicolumn{11}{|c|}{ Others } \\
\hline 131 & Styrene & $100-42-5$ & 1259 & S,MS,RI & 0.219 & 0.254 & 0.690 & 1.039 & 0.532 & 0.790 \\
\hline 132 & $\begin{array}{c}1,2- \\
\text { Dimethoxybenzene }\end{array}$ & $91-16-7$ & 1733 & MS,RI & 0.083 & 0.018 & - & - & - & - \\
\hline 133 & $\begin{array}{c}1,4- \\
\text { Dimethoxybenzene }\end{array}$ & $150-78-7$ & 1747 & S,MS,RI & - & - & - & - & - & 0.026 \\
\hline 134 & Naphthalene & $91-20-3$ & 1750 & S,MS,RI & 0.494 & 0.066 & 0.107 & 0.221 & 0.143 & 0.182 \\
\hline 135 & $\begin{array}{c}1,3- \\
\text { Dimethoxybenzene }\end{array}$ & $151-10-0$ & 1757 & S,MS,RI & 0.145 & 0.066 & 0.123 & 0.020 & 0.093 & 0.074 \\
\hline 136 & 1-Methylnaphthalene & $90-12-0$ & 1861 & S,MS,RI & 0.184 & 0.012 & 0.073 & - & 0.100 & 0.069 \\
\hline 137 & $\begin{array}{l}\text { 3-Ethyl-2-methyl-1,3- } \\
\text { hexadiene }\end{array}$ & $61142-36-7$ & 1418 & MS & 1.717 & - & - & - & - & - \\
\hline 138 & Pentadecane & $629-62-9$ & 1500 & MS,RI & - & 0.045 & 0.090 & 0.160 & 0.126 & 0.162 \\
\hline \multirow[t]{2}{*}{139} & Hexadecane & $544-76-3$ & 1600 & S,MS,RI & - & 0.081 & 0.153 & 0.294 & 0.196 & 0.224 \\
\hline & Subtotal & & & & 2.841 & 0.542 & 1.236 & 1.734 & 1.191 & 1.526 \\
\hline
\end{tabular}

${ }^{a}$ : NIST 11 was used to qualitative analysis, with matching degree $\geq 80$; RI: retention index; S: standard substance was used to qualitative analysis; ${ }^{\mathrm{b}}$ : The area normalization method was used to express the relative content of the compounds. MS: compounds identified by MS spectra.

A

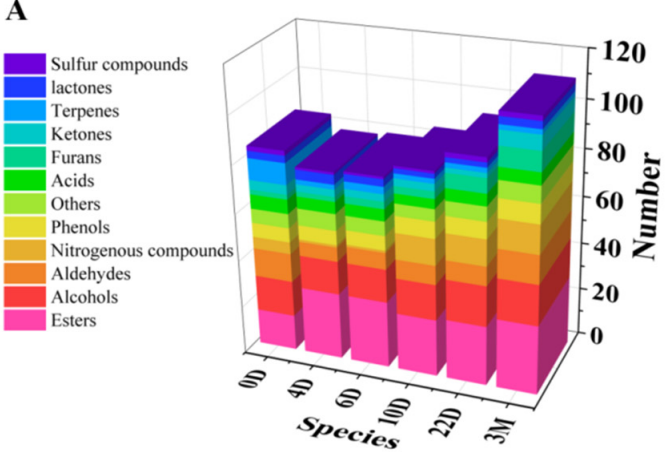

B

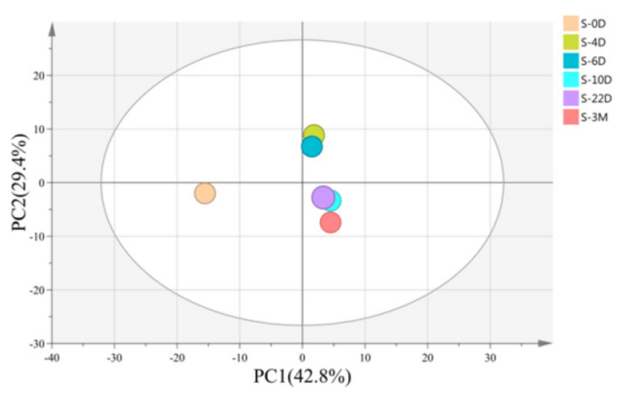

Figure 3. Types and changes of volatile components in Daqu at different maturity stages (A); PCA score plots of different maturity stages Daqu (B). 
Esters, which account for $60 \%$ of all flavor substances in baijiu, play an essential role in baijiu [22]. It can be seen from Table 1 and Figure 3A that the ester compounds in Daqu (S-4D) after early fermentation increased significantly, and the relative peak area reached $43.381 \%$. With the fermentation, the relative peak areas of esters in S-10D, S-22D and S-3M were more than $50 \%$, the kinds of esters increased, and the content of long-chain fatty acid esters such as ethyl pentadecanoate, ethyl octadecenoate, and ethyl hexadecanoate increased significantly.

The relative content of nitrogen-containing substances also increased significantly with the extension of fermentation time. After storage, the range of nitrogen-containing substances in Daqu reached 7.228\%. Nitrogen-containing substances significantly increased after a slow rising period (about S-10D) compared with ester compounds. Among them, the content of tetramethylpyrazine changed the most, which was the most nitrogen-containing compound in Daqu after storage. This is because nitrogen-containing substances were mainly produced by the Maillard reaction. With the fermentation, the fermentation temperature of medium high temperature Daqu could reach $50-60{ }^{\circ} \mathrm{C}$. In the high temperature transformation stage of no less than one week, amino acids and proteins in wheat undergo the Maillard reaction to produce a variety of pyrazines (one of the nitrogenous compounds) [23]. The compounds mainly show a roasting and nut-like aroma, which might contribute to the flavor of the finished Daqu. Nitrogen-containing substances in Daqu might be brought into baijiu.

The number of aldehydes and ketones decreased first and then increased. Daqu used in production is made from wheat, rich in aldehydes and ketones [24]. Therefore, the type and content of aldehydes and ketones in S-0D are relatively high, which might be related to the high content of aldehydes and ketones in grain raw materials. Ten species of aldehydes decreased significantly after the early fermentation (about S-4D) from Table 1. With the progress of fermentation, aldehydes and ketones were generated by non-enzymatic chemical reactions.

Acid compounds are the primary metabolites and the premise substances for the synthesis of other compounds. The changes in the fermentation process are mainly reflected in the changes in the relative content. After the early fermentation (about S-4D), the range of acid compounds reached the maximum (11.086\%) and then decreased slowly. Alcohols and phenols are primary metabolites, showing the same trend of change. The content of alcohols and phenols first increased and then also reduced during Daqu ripening.

Furan compounds have furan heterocyclic structure and strong activity. With the fermentation, the kinds of furan compounds increased significantly, but the overall content of furan compounds decreased. Eight furan compounds were identified in Daqu after storage (S-3M). Furfuryl alcohol content in S-3M increased, which showed coffee aroma, malt aroma, and baking aroma.

Terpenes with isoprene as a structural unit multiple and their oxygen-containing derivatives widely exist in plants [25]. Therefore, the species (9 species) and content (27.399\%) of Daqu (S-0D) were higher, and the species and content decreased significantly after early fermentation.

PCA analysis was performed based on data obtained from area percentage at different maturity stages Daqu in Table 1 to reveal the distribution of samples and detect outliers. PCA score plots of varying maturity stages Daqu were shown in Figure 3B. The first principal component (PC1) accounted for $42.8 \%$ of the variance, and the second principal component (PC2) accounted for $29.4 \%$. Two components (PC1 and PC2) explained 72.2\% of the variance. Different maturity stages Daqu were separated in the PCA score plots. The variables have no outliers and are within the $95 \%$ confidence interval. According to Figure 3B, the S-0D sample was significantly different from the others, and the S-4D and S-6D fermentation samples showed similarities. In addition, S-10D, S-22D, and S-3M have a certain similarity, but S-3M can be appropriately distinguished from the other two samples. 


\subsection{Analysis of Active Aroma Compounds in Daqu S-3M by GC-MS/O}

According to the qualitative results in Table 1, the volatile components of Daqu at different maturity stages are quite different. The greater variety and content of aromas in the Daqu S-3M determined the final use of it in actual production. The following Daqu S-3M was taken as the object to explore the active aroma compounds.

GC-MS/O analyzed the active aroma compounds in Daqu S-3M. A total of 50 aroma regions were smelled under different polarity chromatographic columns, except for seven compounds that were not successfully identified. Forty-three compounds were identified by mass spectrometry, retention index, and standard substance as shown in Table 2: including 8 esters, 2 lactones, 7 alcohols, 8 aldehydes, 5 nitrogenous compounds, 4 phenols and acids, 3 terpenes, 1 sulfur compound, and another compound (naphthalene).

In Table 2, No. 6 (2,6-dimethylpyrazine), No. 8 (dimethyl trisulfide), No. 14 (acetic acid), No. 16 (2-ethyl-3,5-dimethylpyrazine), No. 19 (benzaldehyde) No. 22 ( $\beta$-caryophyllene), No. 24 (butanoic acid), No. 29 (3-methylbutanoic acid), No. 41 (guaiacol), No. 43 (phenethyl alcohol), No. 45 (4-ethyl-2-methoxyphenol), No. 46 ( $\gamma$-nonanolactone), and No. 49 (methyl palmitate) had higher olfactory intensity. In addition, many compounds with low olfactory intensity might affect the overall aroma profile of Daqu [26] because they can have high flavor thresholds.

Esters are the main flavor substances in baijiu, and most of the short-chain esters with the flavor of fruit odor, including No. 2 (ethyl butyrate), No. 3 (ethyl valerate), and No. 5 (ethyl hexanoate). Long-chain fatty acid esters, such as methyl palmitate, have a high threshold but show a significant wax flavor, which may be related to the characteristic aroma of Daqu [23]. There are many types of esters, and their olfactory intensities are relatively low except for No. 49 (methyl palmitate). In addition, among the three lactones identified in S-3M, Daqu, $\gamma$-nonanolactone, and $\gamma$-butyrolactone showed aroma activity, and $\gamma$-nonanolactone with coconut flavor also had high aroma intensity. The results showed that lactones played a significant role in the aroma of baijiu, while $\gamma$-nonanolactone was one of the essential aroma compounds.

A large number of alcohols were identified in this experiment, but the olfactory intensity was low. The smell intensity of phenethyl alcohol and benzyl alcohol containing a benzene ring was relatively high, showing flower, sweet, and honey fragrance.

In addition to No. 44 (2-phenyl-2-butenal, sweet aroma), Daqu also had more aldehydes after storage, which mainly showed a typical compound aroma of grass and oil, bitter almond, and so on. It can be seen from Table 2 that the relative content of No. 44 (2phenyl-2-butenal) among the seven aldehydes identified in Daqu was S-0D $(0.037 \%)<\mathrm{S}-3 \mathrm{M}$ $(0.457 \%)$. In addition, the relative content of nonanal, $(E)$-2-octenal, phenylacetaldehyde, (E)-2-nonenal, phenylacetaldehyde, and 2,4-decadienal is S-0D > S-3M. The compound aroma of these aldehydes has an essential influence on the flavor of Daqu.

Phenol compounds have high odor intensity, which were important aroma active compounds in Daqu. Guaiacol, 4-ethyl-2-methoxyphenol, and 2-methoxy-4-vinylphenol were characterized with wood flavor, clove, scorch bitter taste, etc., while $p$-cresol has an animal odor.

Pyrazine compounds (nitrogenous compounds) are considered the primary source of coke flavor and baking flavor in Daqu [23]. In addition, 2,5-dimethylpyrazine and 2,6-dimethylpyrazine are considered essential aroma compounds in medium and high temperature Daqu. The five pyrazines identified in this experiment are wheat flavor, roasted flavor, and potato flavor.

Dimethyl trisulfide (garlic) and naphthalene (grass) also had higher olfactory intensity in Daqu. Dimethyl trisulfide was considered a vital aroma substance in SAB [6]. According to the above speculation, Daqu and baijiu have common flavor compounds, and they are related on flavor. 
Table 2. Analysis of active aroma compounds in Daqu after storage (S-3M).

\begin{tabular}{|c|c|c|c|c|c|c|c|c|}
\hline \multirow{2}{*}{ No. } & \multirow{2}{*}{ Compounds } & \multirow{2}{*}{$\begin{array}{c}\text { CAS } \\
\text { Number }\end{array}$} & \multirow{2}{*}{$\begin{array}{c}\text { Aroma } \\
\text { Description }\end{array}$} & \multirow{2}{*}{ Identification $^{a}$} & \multicolumn{2}{|c|}{ RI } & \multicolumn{2}{|c|}{ Olfactory Intensity } \\
\hline & & & & & DB-WAX $^{\mathbf{b}}$ & $\mathrm{HP}-5^{\mathrm{c}}$ & DB-WAX & HP-5 \\
\hline 1 & Ethyl 3-methylbutanoate & $108-64-5$ & fruity & MS, aroma, RI, S & 1071 & 859 & 2.2 & 2.0 \\
\hline 2 & Ethyl butyrate & $105-54-4$ & fruity & MS, aroma, RI, S & 1026 & - & 1.3 & - \\
\hline 3 & Ethyl valerate & $539-82-2$ & fruity & MS, aroma, RI, S & 1142 & 898 & 1.0 & 3.5 \\
\hline 4 & Limonene & $138-86-3$ & sour/sweat & MS, aroma, RI, S & 1189 & - & 2.7 & - \\
\hline 5 & Ethyl hexanoate & $123-66-0$ & fruity & MS, aroma, RI, S & 1240 & 1001 & 2.5 & 3.0 \\
\hline 6 & 2,6-Dimethylpyrazine & $108-50-9$ & wheat/roasted & MS, aroma, RI, S & 1347 & - & 4.0 & - \\
\hline 7 & 2,3-Dimethylpyrazine & $5910-89-4$ & wheat/cocoa & MS, aroma, RI, S & 1349 & 922 & - & 3.7 \\
\hline 8 & Dimethyl trisulfide & $3658-80-8$ & garlic & MS, aroma, RI, S & 1370 & 975 & 4.0 & 3.8 \\
\hline 9 & $\begin{array}{l}\text { 3-Ethyl-2- } \\
\text { methylpyrazine }\end{array}$ & 15707-23-0 & roasted & MS, aroma, RI, S & 1395 & - & 1.0 & - \\
\hline 10 & Nonanal & $124-19-6$ & oily & MS, aroma, RI, S & 1396 & 1108 & - & 3.0 \\
\hline 11 & 2,3,5-Trimethylpyrazine & $14667-55-1$ & earthy & MS, aroma, RI, S & 1410 & - & 3.3 & - \\
\hline 12 & 2-Octanol & $123-96-6$ & soapy/creamy & MS, aroma, RI, S & 1412 & - & 3.5 & - \\
\hline 13 & (E)-2-Octenal & $2548-87-0$ & grassy/fatty & MS, aroma, RI, S & 1429 & - & 2.5 & - \\
\hline 14 & Acetic acid & 64-19-7 & acetic & MS, aroma, RI, S & 1452 & - & 4.0 & - \\
\hline 15 & 1-Heptanol & $111-70-6$ & grassy & MS, aroma, RI, S & 1452 & 980 & - & 2.5 \\
\hline 16 & $\begin{array}{c}\text { 2-Ethyl-3,5- } \\
\text { dimethylpyrazine }\end{array}$ & $13925-07-0$ & boiled potato & MS, aroma, RI, S & 1464 & 1085 & 4.0 & 2.5 \\
\hline 17 & & & herbal & aroma & 1480 & - & 1.0 & - \\
\hline 18 & 2-Ethylhexanol & $104-76-7$ & grassy & MS, aroma, RI, S & 1489 & - & 1.0 & - \\
\hline 19 & Benzaldehyde & $100-52-7$ & bitter almond & MS, aroma, RI, S & 1508 & 961 & 3.7 & 2.0 \\
\hline 20 & (E)-2-Nonenal & $18829-56-6$ & grassy/fatty & MS, aroma, RI, S & 1536 & - & 2.5 & - \\
\hline 21 & & & minty & aroma & 1574 & - & 2.5 & - \\
\hline 22 & $\beta$-Caryophyllene & $87-44-5$ & woody & MS, aroma, RI, S & 1585 & - & 3.8 & - \\
\hline 23 & & & wheat & aroma & 1602 & - & 2.5 & - \\
\hline 24 & Butanoic acid & $107-92-6$ & sour and stinky & MS, aroma, RI, S & 1628 & - & 4.0 & - \\
\hline 25 & $\gamma$-Butyrolactone & $96-48-0$ & wheat & MS, aroma, RI, S & 1643 & - & 2.3 & - \\
\hline 26 & Phenylacetaldehyde & $122-78-1$ & penicillin/grassy & MS, aroma, RI, S & 1654 & 1044 & - & 3.0 \\
\hline 27 & Furfuryl alcohol & $98-00-0$ & burnt/caramel & MS, aroma, RI, S & 1666 & - & 1.7 & - \\
\hline 28 & Ethyl benzoate & $93-89-0$ & musty & MS, aroma, RI, S & 1672 & 1173 & 2.0 & 2.5 \\
\hline 29 & 3-Methylbutanoic acid & $503-74-2$ & sour and stinky & MS, aroma, RI, S & 1680 & - & 4.0 & - \\
\hline 30 & Diethyl succinate & $123-25-1$ & wheat/fruity & MS, aroma, RI, S & 1704 & - & 1.0 & - \\
\hline 31 & Naphthalene & $91-20-3$ & grassy & MS, aroma, RI, S & 1750 & 1182 & - & 3.8 \\
\hline 32 & & & herbal(powdery) & aroma & 1760 & - & 3.8 & - \\
\hline 33 & Ethyl phenylacetate & $101-97-3$ & sweety & MS, aroma, RI, S & 1793 & 1247 & - & 2.0 \\
\hline 34 & 2,4-Decadienal & $25152-84-5$ & fatty/grassy & MS, aroma, RI, S & 1818 & 1316 & 2.0 & 2.2 \\
\hline 35 & 1-Phenylethanol & $98-85-1$ & vanilla & MS, aroma, RI, S & 1820 & - & 1.5 & - \\
\hline 36 & & & wheat & aroma & 1832 & - & 2.0 & - \\
\hline 37 & Geranyl acetone & $3796-70-1$ & grassy & MS, aroma, RI, S & 1856 & 1453 & - & 2.5 \\
\hline 38 & & & bitter & aroma & 1857 & 1349 & 3.3 & 3.2 \\
\hline 39 & & & sweet & aroma & 1862 & 1390 & 3.0 & 3.5 \\
\hline 40 & Hexanoic acid & $142-62-1$ & sour & MS, aroma, RI, S & 1866 & - & 3.2 & - \\
\hline 41 & Guaiacol & $90-05-1$ & woody & MS, aroma, RI, S & 1871 & 1096 & 4.0 & 3.0 \\
\hline 42 & Benzyl alcohol & $100-51-6$ & sweet/floral & MS, aroma, RI, S & 1877 & - & 2.5 & - \\
\hline 43 & Phenethyl alcohol & $60-12-8$ & honey & MS, aroma, RI, S & 1901 & 1114 & 4.0 & 4.0 \\
\hline 44 & 2-Phenyl-2-butenal & $4411-89-6$ & sweet & MS, aroma, RI, S & 1917 & - & 3.1 & - \\
\hline 45 & $\begin{array}{c}\text { 4-Ethyl-2- } \\
\text { methoxyphenol }\end{array}$ & $2785-89-9$ & clove & MS, aroma, RI, S & 2018 & 1279 & 4.0 & 3.5 \\
\hline 46 & $\gamma$-Nonanolactone & $104-61-0$ & coconut & MS, aroma, RI, S & 2020 & 1368 & 4.0 & 2.8 \\
\hline 47 & $p$-Cresol & $106-44-5$ & animal & MS, aroma, RI, S & 2078 & - & 3.0 & - \\
\hline 48 & $\begin{array}{l}\text { 2-Methoxy-4- } \\
\text { vinylphenol }\end{array}$ & $7786-61-0$ & bake/bitter & MS, aroma, RI, S & 2184 & 1309 & 3.2 & 1.2 \\
\hline 49 & Methyl palmitate & $112-39-0$ & wax & MS, aroma, RI, S & 2218 & 1929 & 4.0 & 3.9 \\
\hline 50 & Hexanal & $66-25-1$ & fatty/grassy & MS, aroma, RI, S & 1078 & 838 & 3.1 & 2.0 \\
\hline
\end{tabular}

a: NIST 11 was used to qualitative analysis, with matching degree $\geq 80$; RI: retention index; S: standard substance was used to qualitative analysis; ${ }^{b}$ : Linear retention index of DB-wax; ${ }^{c}$ : Linear retention index of HP-5.

\subsection{Quantitative Analysis of Aroma Components in Daqu by MHS-SPME}

The quantitative results of 21 compounds in Daqu have been reported in a previous article [21]. Quantitative Results showed the data of the standard curve obtained by MHSSPME: the correlation coefficient $\left(\mathrm{R}^{2}\right)$ is between 0.9717 and 0.9983 , and the fitting is good. The LOD of all compounds was less than $68.4 \mathrm{ppb}$, which showed high sensitivity. The RSD of the compounds was calculated using the results of three replicates. The RSD of each 
compound was lower than $11.75 \%$. The experimental repeatability was good at the same time. The recoveries of the compounds ranged from $83.58 \%$ to $120.05 \%$, which met the needs of experimental quantification. In the quantitative analysis, the content range was $12.93 \mathrm{ng} / \mathrm{g}-2816.28 \mathrm{ng} / \mathrm{g}$. The content of furfuryl alcohol (2816.28 ng/g) with the burned taste was the highest. In addition, the content of phenylacetaldehyde, guaiacol, nonanal, and $\gamma$-butyrolactone was higher than $500 \mathrm{ng} / \mathrm{g}$. The contents of 2-phenyl-2-butenal, 2ethyl-3,5-dimethylpyrazine, geranyl acetone, and 3-ethyl-2-methylpyrazine were lower than $100 \mathrm{ng} / \mathrm{g}$.

The content of the compound only indicates the amount of the compound in the sample, which can not reflect the effect of the compound on the overall flavor of the sample. For example, furfuryl alcohol had the highest content, but its olfactory intensity was low. Therefore, based on quantitative analysis, combined with the threshold value of compounds, OAV value was calculated to evaluate the effect of different compounds on the overall flavor of samples [26].

The OAV values of 21 compounds were calculated as shown in Table 3 , and 18 compounds OAVs $\geq 1$, which contributed greatly to the overall aroma of Daqu and were important flavor compounds in Daqu aroma. In exploring Baijiu flavor, grain aroma is often described as one of the flavor descriptors and is an important flavor branch of baijiu flavor quality evaluation. Nine compounds related to "grain aroma" have higher OAV values except for geranyl acetone (OAV = 1). (E,E)-2,4-decadienal has the highest OAV value $(\mathrm{OAV}=2746)$ among them. $(E, E)-2,4$-decadienal $(\mathrm{OAV}=2746)$, nonanal $(\mathrm{OAV}=655)$, phenylacetaldehyde (OAV = 447), (E)-2-nonenal (OAV = 339), hexanal (OAV = 81), 4-ethyl2-methoxyphenol $(\mathrm{OAV}=40),(E)$-2-octenal $(\mathrm{OAV}=40)$, and $\gamma$-nonanolactone $(\mathrm{OAV}=10)$ have an important contribution to Daqu aroma. It was consistent with other literature that nonanal and 4-ethyl-2-methoxyphenol were considered aroma active substances in Daqu [26].

Table 3. OAV calculation for the quantitative compounds.

\begin{tabular}{|c|c|c|c|}
\hline No. & Compounds & Threshold a/(ppb) & OAVs \\
\hline 6 & 2,6-Dimethylpyrazine & 200 & 2 \\
\hline 7 & 2,3-Dimethylpyrazine & 10823.7 & $<1$ \\
\hline 9 & 3-Ethyl-2-methylpyrazine & 130 & $<1$ \\
\hline 10 & Nonanal & 1 & 655 \\
\hline 11 & 2,3,5-Trimethylpyrazine & 10 & 24 \\
\hline 13 & (E)-2-Octenal & 3 & 40 \\
\hline 16 & 2-Ethyl-3,5-dimethylpyrazine & 1 & 43 \\
\hline 19 & Benzaldehyde & 30 & 5 \\
\hline 20 & (E)-2-Nonenal & 0.6 & 339 \\
\hline 22 & $\beta$-Caryophyllene & 64 & 2 \\
\hline 25 & $\gamma$-Butyrolactone & 50 & 11 \\
\hline 26 & Phenylacetaldehyde & 4 & 447 \\
\hline 27 & Furfuryl alcohol & 100 & 28 \\
\hline 34 & 2,4-Decadienal & 0.07 & 2746 \\
\hline 37 & Geranyl acetone & 60 & 1 \\
\hline 41 & Guaiacol & 5.5 & 172 \\
\hline 44 & 2-Phenyl-2-butenal & - & - \\
\hline 45 & 4-Ethyl-2-methoxyphenol & 6.9 & 40 \\
\hline 46 & $\gamma$-Nonanolactone & 21 & 10 \\
\hline 48 & 2-Methoxy-4-vinylphenol & 3 & 122 \\
\hline 50 & Hexanal & 25.48 & 81 \\
\hline
\end{tabular}

a: Check the threshold of the corresponding compound on http://www.leffingwell.com (accessed on 23 July 2020).

In addition, eight compounds had $\mathrm{OAV} \geq 1$. Among them, guaiacol (OAV = 172), 2-methoxy-4-vinylphenol (OAV = 122), 2-ethyl-3,5-dimethylpyrazine (OAV = 43), furfuryl 
alcohol (OAV = 28), 2,3,5-trimethylpyrazine (OAV = 24), 2-ethyl-3,5-dimethylpyrazine $(\mathrm{OAV}=22)$, and $\gamma$-butyrolactone $(\mathrm{OAV}=11)$ were higher.

\section{Conclusions}

Under the optimal extraction conditions, 139 compounds are identified in the six different maturity stages of Daqu. Through the analysis of the variety of compounds and the PCA analysis of the compound contents, the scientific basis for Daqu S-3M widely used in practical production is provided from the flavor perspective. In the maturing process of Daqu, ester compounds were mainly produced in the early stage of fermentation, and pyrazine compounds were mainly produced after the slow rising stage, and the content and species were relatively stable after that. The number of aldehydes and ketones decreased first and then increased. The content of acids reached the maximum after early fermentation and then decreased slowly. The contents of alcohols and phenols increased at first and then decreased during Daqu ripening. The kinds of furan compounds increased with the fermentation. The types and contents of terpenoids decreased obviously after early fermentation.

In this experiment, HS-SPME combined with GC-MS/O was used to analyze the active aroma substances in the Daqu S-3M, and 42 active aroma compounds were identified. Among the 21 aroma compounds quantitatively analyzed, 18 compounds with OAV $\geq 1$ were important in Daqu aroma including guaiacol, 4-ethyl-2-methoxy phenol, 2-ethyl-3,5dimethylpyrazine, furfuryl alcohol, 2,3,5-trimethylpyrazine, etc. It provides a reference for the study of Daqu from the perspective of flavor. This provides a scientific basis for the use of Daqu in practice.

Supplementary Materials: The following supporting information can be downloaded at: https: / / www.mdpi.com/article/10.3390/foods11010116/s1. Figure S1 Baijiu flavour wheel [5]; Figure S2 TIC diagram of Daqu after storage (S-3M); Table S1 Sample code and status.

Author Contributions: Z.W.: Methodology, Software, Writing—original draft, Writing—review and editing; S.W.: Investigation, Methodology, Writing —original draft; P.L.: Investigation, Software; L.C.: Investigation, Software; J.S.: Writing-review and editing, Supervision; B.S.: Writing-review and editing, Supervision; D.Z.: Writing-review and editing, Supervision; B.W.: Writing-review and editing, Supervision; H.L.: Writing-review and editing, Supervision. All authors have read and agreed to the published version of the manuscript.

Funding: The research was funded by the National Natural Science Foundation of P. R. China (No. 31972193); Science and Technology program of Tibet Autonomous Region, China (XZ202001ZY0017N).

Institutional Review Board Statement: Not applicable.

Informed Consent Statement: Not applicable.

Data Availability Statement: Date of the compounds are available from the authors.

Acknowledgments: Gujing Group Co., Ltd. provided samples for this experiment.

Conflicts of Interest: The authors declare that they have no known competing financial interests or personal relationships that could have appeared to influence the work reported in this paper.

\section{References}

1. Liu, H.; Sun, B. Effect of Fermentation Processing on the Flavor of Baijiu. J. Agric. Food Chem. 2018, 66, 5425-5432. [CrossRef]

2. Jin, G.; Zhu, Y.; Xu, Y. Mystery behind Chinese liquor fermentation. Trends Food Sci. Technol. 2017, 63, 18-28. [CrossRef]

3. Wang, B.; Wu, Q.; Xu, Y.; Sun, B. Synergistic Effect of Multiple Saccharifying Enzymes on Alcoholic Fermentation for Chinese Baijiu Production. Appl. Environ. Microbiol. 2020, 86, e00013-20. [CrossRef] [PubMed]

4. Sun, J.; Wang, Z.; Sun, B. Low Quantity but Critical Contribution to Flavor: Review of The Current Understanding of Volatile Sulfur-containing Compounds in Baijiu. J. Food Compos. Anal. 2021, 103, 104079. [CrossRef]

5. GB/T 33405-2016; Terminology of Baijiu Sensory Evaluation. National Technical Committee 358 on Chinese Spirits of Standardization Administration of China: Beijing, China, 2016. 
6. Zhao, D.; Shi, D.; Sun, J.; Li, A.; Sun, B.; Zhao, M.; Chen, F.; Sun, X.; Li, H.; Huang, M.; et al. Characterization of key aroma compounds in Gujinggong Chinese Baijiu by gas chromatography-olfactometry, quantitative measurements, and sensory evaluation. Food Res. Int. 2018, 105, 616-627. [CrossRef] [PubMed]

7. He, F.; Duan, J.; Zhao, J.; Li, H.; Sun, J.; Huang, M.; Sun, B. Different distillation stages Baijiu classification by temperatureprogrammed headspace-gas chromatography-ion mobility spectrometry and gas chromatography-olfactometry-mass spectrometry combined with chemometric strategies. Food Chem. 2021, 365, 130430. [CrossRef] [PubMed]

8. Dong, W.; Guo, R.; Liu, M.; Shen, C.; Sun, X.; Zhao, M.; Sun, J.; Li, H.; Zheng, F.; Huang, M.; et al. Characterization of key odorants causing the roasted and mud-like aromas in strong-aroma types of base Baijiu. Food Res. Int. 2019, 125, 108546. [CrossRef]

9. Costa, R.; Tedone, L.; De Grazia, S.; Dugo, P.; Mondello, L. Multiple headspace-solid-phase microextraction: An application to quantification of mushroom volatiles. Anal. Chim. Acta 2013, 770, 1-6. [CrossRef] [PubMed]

10. Rigling, M.; Fraatz, M.A.; Trögel, S.; Sun, J.; Zorn, H.; Zhang, Y. Aroma Investigation of Chios Mastic Gum (Pistacia lentiscus Variety Chia) Using Headspace Gas Chromatography Combined with Olfactory Detection and Chiral Analysis. J. Agric. Food Chem. 2019, 67, 13420-13429. [CrossRef]

11. Serrano, E.; Beltrán, J.; Hernández, F. Application of multiple headspace-solid-phase microextraction followed by gas chromatography-mass spectrometry to quantitative analysis of tomato aroma components. J. Chromatogr. A 2009, 1216, 127-133. [CrossRef]

12. Rincón, A.A.; Pino, V.; Ayala, J.H.; Afonso, A.M. Multiple headspace solid-phase microextraction for quantifying volatile free fatty acids in cheeses. Talanta 2014, 129, 183-190. [CrossRef] [PubMed]

13. Flores, M.; Hernandez, D. Optimization of multiple headspace solid-phase microextraction for the quantification of volatile compounds in dry fermented sausages. J. Agric. Food Chem. 2007, 55, 8688-8695. [CrossRef] [PubMed]

14. Carrillo, J.D.; Tena, M.T. Determination of volatile oak compounds in aged wines by multiple headspace solid-phase microextraction and gas chromatography-mass spectrometry (MHS-SPME-GC-MS). Anal. Bioanal. Chem. 2006, 385, 937-943. [CrossRef]

15. Pizarro, C.; Pérez-Del-Notario, N.; González-Sáiz, J. Determination of Brett character responsible compounds in wines by using multiple headspace solid-phase microextraction. J. Chromatogr. A 2007, 1143, 176-181. [CrossRef]

16. Ye, C.; Zhang, X.; Huang, J.; Li, S.; Pan, S.; Wang, Y.; Li, X. Multiple headspace solid-phase microextraction of ethyl carbamate from different alcoholic beverages employing drying agent based matrix modification. J. Chromatogr. A 2011, 1218, 5063-5070. [CrossRef]

17. Rega, B.; Fournier, N.; Guichard, E. Solid phase microextraction (SPME) of orange juice flavor: Odor representativeness by direct gas chromatography olfactometry (D-GC-O). J. Agric. Food Chem. 2003, 51, 7092-7099. [CrossRef]

18. Feng, Y.; Su, G.; Sun-Waterhouse, D.; Cai, Y.; Zhao, H.; Cui, C.; Zhao, M. Optimization of Headspace Solid-Phase Microextraction (HS-SPME) for Analyzing Soy Sauce Aroma Compounds via Coupling with Direct GC-Olfactometry (D-GC-O) and Gas Chromatography-Mass Spectrometry (GC-MS). Food Anal. Methods 2016, 10, 713-726. [CrossRef]

19. Shi, K.; Sun, X.; Shen, C.; Ao, L.; Zheng, F.; Huang, M.; Sun, J.; Li, H. Study on the key aroma components of Luzhou-flavor baijiu based on overall sensory evaluation model by direct-gas chromatography-olfaction. Sci. Technol. Food Ind. 2020, 41, 208-219.

20. Sha, S.; Chen, S.; Qian, M.; Wang, C.; Xu, Y. Characterization of the Typical Potent Odorants in Chinese Roasted Sesame-like Flavor Type Liquor by Headspace Solid Phase Microextraction-Aroma Extract Dilution Analysis, with Special Emphasis on Sulfur-Containing Odorants. J. Agric. Food Chem. 2017, 65, 123-131. [CrossRef]

21. Wang, S.; Chen, L.; Liu, G.; Sun, J.; Zhang, Y.; Li, H.; Sun, B. Application of multiple headspace solid phase microextraction in quantification of volatile compounds in daqu. Fine Chem. 2020, 37, 346-355+390.

22. Xu, Y.; Zhao, J.; Liu, X.; Zhang, C.; Zhao, Z.; Li, X.; Sun, B. Flavor mystery of Chinese traditional fermented baijiu: The great contribution of ester compounds. Food Chem. 2022, 369, 130920. [CrossRef] [PubMed]

23. Zheng, J.; Peng, Z.; Zhao, D. Research Progress in Flavor Chemistry of Traditional Daqu. Liquor-Mak. Sci. Technol. 2017, 89-94.

24. Hu, D.; Zhang, Y.; Zhang, T.; Hou, J.; Ren, Y.; Chen, C. Analysis of Volatile Aroma Components in Baked Wheat Germ by Simultaneous Distillation and Extraction or Dynamic Headspace Extraction Coupled with GC-MS. Food Sci. 2012 , 33, $236-242$.

25. Xu, Y.; Fan, W.; Ge, X.; Huang, Y. Scientific Recognition of Biofunctional Components in Chinese Liquors. Liquor-Mak. Sci. Technol. 2013, 9, 1-6.

26. Zhang, C.; Ao, Z.; Chui, W.; Shen, C.; Tao, W.; Zhang, S. Characterization of the aroma-active compounds in Daqu: A tradition Chinese liquor starter. Eur. Food Res. Technol. 2012, 234, 69-76. [CrossRef] 